\title{
Considering the Impact of Type 2 Diabetes Mellitus-Biological Mechanisms in COVID-19 Pathology
}

\author{
Debasree Banerjee, ${ }^{1}$ Morgan R Askew, ${ }^{2}$ and Andrew H Stephen ${ }^{3}$ \\ 1. Department of Medicine, Alpert Medical School of Brown University, Providence, RI, USA; 2. Alpert Medical School of Brown University, Providence, \\ RI, USA; 3. Department of Surgery, Alpert Medical School of Brown University, Providence, RI, USA
}

DOI: https://doi.org/10.17925/USPRD.2020.5.1.24

$\mathrm{D}$ iabetes is one of the most prevalent comorbidities among patients with COVID-19, and specific minorities in the USA are being disproportionately affected. The biologic mechanism underpinning this association is still unknown; however, there are known causal relationships between hyperglycemia and dysregulated immunity in the diabetic population in sepsis and acute respiratory distress syndrome. Diabetes may impact the pathogenicity of severe acute respiratory syndrome coronavirus 2 (SARS-CoV-2), possibly via glycosylation of a key receptor, angiotensin-converting enzyme 2. It is imperative that we further understand this link in order to attenuate the critical illness caused by COVID-19 among diabetics.

\section{Keywords}

Angiotensin-converting enzyme 2 , COVID-19, diabetes

Disclosures: Debasree Banerjee, Morgan R Askew, and Andrew $\mathrm{H}$ Stephen have no financial or non-financial relationships or activities to declare in relation to this article. Review Process: Double-blind peer review.

Compliance with Ethics: This article involves a review of the literature and did not involve any studies with human or animal subjects performed by any of the authors.

Authorship: The named authors meet the International Committee of Medical Journal Editors (ICMJE) criteria for authorship of this manuscript, take responsibility for the integrity of the work as a whole, and have given final approval for the version to be published.

Access: This article is freely accessible at touchRESPIRATORY.com (c) Touch Medical Media 2020.

Received: June 5, 2020

Accepted: October 5, 2020

Published Online: December 23, 2020

Citation: US Respiratory \& Pulmonary Diseases. 2020; 5(1):24-7

Corresponding Author: Andrew H Stephen, Division of Trauma and Surgical Critical Care, Department of Surgery, Alpert Medical School of Brown University, 435 APC

Building, 4th Floor, 593 Eddy Street, Rhode Island Hospital,

Providence, RI 02903, USA.

E: andrew_stephen@brown.edu

Support: No funding was received in

the publication of this article.
The COVID-19 pandemic has infected more than 61 million people and led to more than 1 million deaths worldwide. ${ }^{1}$ The USA was one of the later countries to experience large scale community transmission; to date, there have been 13.2 million cases and approaching 300,000 deaths attributed to COVID-19. The USA has the highest overall deaths globally. ${ }^{1,2}$ The COVID-19 pandemic has highlighted the extreme healthcare disparities present among the diverse population of the USA. It is important that we identify the populations and patient characteristics that are associated with poor prognostic factors, such as intensive care unit (ICU) admission, need for mechanical ventilation, and mortality, in order to efficiently mobilize resources and to potentially mitigate these negative outcomes. Our local experience at Rhode Island Hospital, Providence, RI, USA, points towards a link between the presence of diabetes mellitus (DM) and glycated hemoglobin ( $\mathrm{HBA} 1 \mathrm{C})>6.5 \%$ and poor outcomes among adult patients with COVID-19 in the ICU. For the purpose of this review, we will refer to observed associations between type 2 DM (T2DM) and COVID-19, though most original publications did not differentiate between types 1 and 2 DM.

DM portends worse outcome in sepsis and respiratory failure, making it vital that we understand the epidemiology and plausible biologic mechanisms underlying observed increased fatalities among diabetics with COVID-19, the deadliest pandemic in the USA in the last century. In an effort to increase access to real-time data, medical journals have removed restrictions for non-subscribers and accelerated their publishing process including limiting use of the time-consuming peer-review process. ${ }^{3}$ This has allowed for a rapid accumulation of patient data for providers to access during the COVID-19 pandemic. Our goal is to explore the role of DM in COVID-19 by first considering data and mechanisms from prior studies of patients with diabetes with critical illness, and then to review published outcomes during the current pandemic.

We searched PubMed with the following keywords: "diabetes", "COVID-19", "SARS-CoV-2", "ACE2", and "hyperglycemia", to explore internationally reported findings from November 1, 2019 to May 30, 2020. References from original articles were then also surveyed, if not captured in the above query.

\section{Diabetes mellitus and outcomes in critical illness -Prior pandemic coronaviruses}

DM is known to alter the immune response to infection, dampening neutrophil chemotaxis, phagocytosis and intracellular killing of pathogens. ${ }^{4}$ While there are no specific guidelines for the diagnosis of DM in the critically ill, hyperglycemia is common among ICU patients and can increase mortality among patients with and without diabetes. ${ }^{5} \mathrm{~A}$ landmark meta-analysis identified DM and hyperglycemia after myocardial infarction to be significantly associated with an increased risk of death 
and the development of congestive heart failure and shock. ${ }^{5}$ Hyperglycemia also increases the risk of infection postoperatively, ${ }^{6}$ and concordantly, effective glycemic control can decrease infection risk after cardiac surgery. Intensive glucose control protocols correlate with decreased markers of inflammation in patients in the ICU. ${ }^{8}$ Infections, particularly pneumonia, are an important cause of mortality for patients with DM, and DM increases the risk of complications, such as hospitalization and ICU admission from viral respiratory illness. ${ }^{9} 10$ The odds of ICU admission were more than quadrupled in a review of almost 250 hospitalized patients with H1N1 influenza. ${ }^{10}$

Prior outbreaks of coronaviruses, such as severe acute respiratory syndrome (SARS) and Middle East respiratory syndrome (MERS), affected patients with DM disproportionately, ${ }^{1,12}$ and were associated with higher mortality in this group. ${ }^{13}$ Retrospective analyses of patients with SARS concluded that a history of DM and hyperglycemia in the hospital were independent risk factors for death, 14,15 and that glucose levels correlated with hypoxemia. ${ }^{15}$ In one series of patients with SARS in the greater Toronto area, DM was associated with greater relative risk of mortality and need for ICU admission or mechanical ventilation, than any other identified pre-illness comorbidities. ${ }^{12}$ Patients with DM and those of advanced age have also suffered worse outcomes from MERS. A small review of hospitalized patients with MERS in Saudi Arabia found a series of lab derangements on presentation such as low lymphocyte count, high neutrophil count, and high serum creatinine to be associated with ICU admission and death, but DM was the only comorbidity associated with these adverse outcomes. ${ }^{16} \mathrm{~A}$ limited pathologic examination comparing a patient with diabetes and one without, both with MERS, demonstrated elevated viral titers systemically in the patient with DM. ${ }^{17}$ Given the massive and growing burden of DM globally, and the wider spread of the COVID-19 pandemic compared with SARS and MERS, the effects of DM on outcomes will likely be much more profound.

\section{Diabetes mellitus epidemiology in COVID-19 and diabetes as a risk factor}

The USA has the highest rate of DM (11\%) out of the 37 countries in the developed nations, among adults aged 20-79 years. ${ }^{18}$ In the USA, early descriptive literature has identified trends in COVID-19 patient characteristics, including high rates of DM in those who require hospital admission for COVID-19.19 In our experience thus far, in a tertiary care hospital system in Southern New England, we have found that patients with COVID-19 are often hyperglycemic with refractory glucose levels, which may be due both to the critical illness stress response and the underlying insulin resistance. Many patients without a formal diagnosis of DM on admission have had DM-range HbA1C ( $26.5 \%)$ levels. These were checked based on observed hyperglycemia or a patient's presumed high adipose mass to lean body mass ratio. ${ }^{20}$

DM has been a common comorbidity among hospitalized patients with COVID-19 infection, 21,22 including in some of the earliest reported case series in China. ${ }^{23}$ It has been suggested that prolonged uncontrolled hyperglycemia, and not just a history of DM, may be important in the pathogenesis of the disease. ${ }^{24}$ Although T1DM is a greater risk factor for blood glucose variability and diabetic ketoacidosis (characterized by uncontrolled hyperglycemia), ${ }^{25}$ only $5.6 \%$ of all patients with DM have the autoimmune T1DM. ${ }^{26}$ Though SARS-CoV-2 may represent a threat to patients with T1DM, more research is needed to understand if SARS-COV-2 disproportionately impacts hyperglycemic patients with T1DM or T2DM..$^{27,28}$
Figure 1: Proposed paracrine loop between the pancreas and lungs in SARS-CoV-2 infection among patients with diabetes

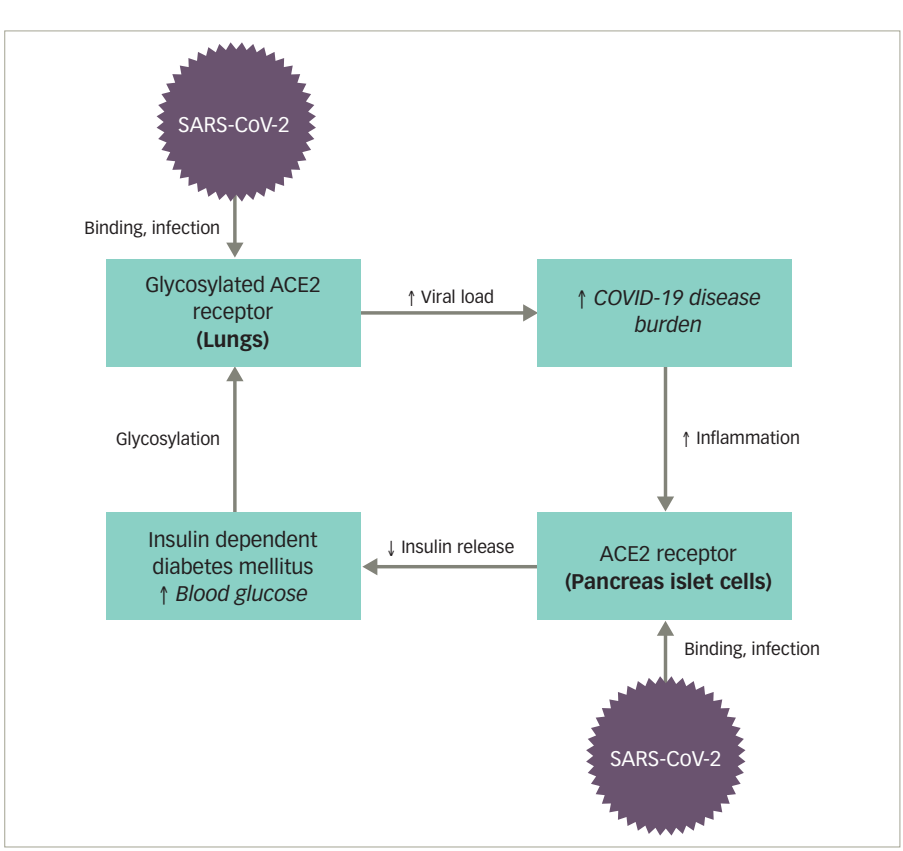

ACE2 = angiotensin-converting enzyme 2.

One meta-analysis that included six studies and 1,558 patients showed that DM was a risk factor for developing COVID-19 infection requiring hospital admission, ${ }^{29}$ another showed DM, hypertension, and other cardiovascular disease, to be the most common comorbidities in hospitalized patients. ${ }^{30}$ While the precise prevalence of DM among COVID-19 cases is unknown, rates of DM in patients with COVID-19 in China and Italy, two of the early global epicenters, were either equal to or greater than rates seen in the general population and were associated with worse outcomes. ${ }^{31}$ DM was present in up to $15 \%$ of hospitalized patients with COVID-19 in China ${ }^{32}$ and up to $35 \%$ of those who died in an Italian cohort. ${ }^{33}$ An analysis of 168 patients who died in China revealed that DM was the second most common comorbidity after hypertension. ${ }^{34}$ In New York City, DM was present in 15\% of hospitalized patients; again, a higher rate than in the general population. ${ }^{35}$ The rate of DM in severe COVID-19 cases requiring ICU admission was twice that of non-ICU cases in one cohort, and another early observational study showed that patients with diabetes are more likely to require ICU level of care. ${ }^{36,37}$ In one of the first case series describing community spread of SARS-COV-2 in the USA, DM was by far the most common comorbidity among hospitalized patients, present in more than half of patients (58\%), followed by positive smoking status (22\%). ${ }^{38}$ Most patients were admitted from home and only half had a known sick contact, offering some implication that DM may be a risk factor for developing symptomatic disease.

In a more recent investigation in the USA, through the end of March 2020, $10 \%$ of a random sampling of COVID-19 cases had DM, with rates higher among those with worse disease severity: $6 \%$ in non-hospitalized patients, $24 \%$ in hospitalized ward-level patients, and $32 \%$ in the critically ill. ${ }^{39}$ DM has also been shown to be associated with a need for mechanical ventilation and increased mortality. ${ }^{40} \mathrm{~A}$ prospective observational study of 174 patients with COVID-19 revealed that those with DM had more severe pneumonia and higher levels of inflammatory and procoagulant biomarkers, including 
interleukin-6, ferritin, C-reactive protein, and D dimer, compared with non-diabetics. ${ }^{41}$ It is important to consider that the diagnosis of DM may not encompass all patients who are at a higher risk of mortality from SARS-CoV-2 due to hyperglycemia. One-third of patients with T2DM are undiagnosed and it is common for the diagnosis to be first made when a patient is cared for in an ICU for sepsis or another illness. ${ }^{20} \mathrm{~A}$ retrospective study at two hospitals in Wuhan also identified a fasting blood glucose $\geq 126 \mathrm{mg} / \mathrm{dL}$, present in $45.6 \%$ of patients, even with no prior DM diagnosis, as an independent predictor of 28 -day mortality. ${ }^{42}$

\section{Mechanisms to explain diabetes mellitus-related exacerbation of COVID-19 illness}

Mouse models of MERS demonstrate that diabetic mice have a protracted hypoinflammatory state irrespective of viral load.43 Transgenic mice expressing humanized MERS receptors in lung epithelium were fed a high-fat diet to mimic the T2DM phenotype. These mice were subsequently infected with the MERS virus and followed for 21 days. Diabetic mice suffered a longer and more severe disease course with fewer CD4+ T cells and macrophages recruited to the lungs initially compared with control mice. Concordantly, diabetic mice expressed lower regional levels of chemokines CCL2 and CXCL10 in the lungs. These mice also had unresolved inflammation on lung histology at the end of the observed period compared with controls, indicating that DM may have a role in causing an altered immune response leading to increased morbidity.

DM was part of a composite marker of angiotensin-converting enzyme 2 (ACE2) associated factors, along with hypertension and other cardiovascular diseases that were associated with delayed viral clearance in a chinese cohort of patients with COVID-19.24 The authors of this pre-print postulate that DM and cardiovascular disease may upregulate expression of ACE2 allowing increased entry of SARS-CoV-2 into type 2 pneumocytes, which are abundant in ACE2 receptors. SARS-CoV and SARS-COV-2 have been shown to mainly utilize the ACE2 receptor for cellular entry. ${ }^{44}$ In vivo studies of severe influenza models have also shown that $\mathrm{T}$ cells lacking insulin receptor activity have blunted antigen responses. ${ }^{45}$ The diminished ability of these $\mathrm{T}$ cells to proliferate and mount production of pro-inflammatory cytokines support a concept of immunometabolism, which may have significance with other respiratory viral illnesses including SARS-CoV-2.

Other authors have promoted the connection between DM and higher ACE2 levels. In a large genome-wide association study, DM and early commencement of insulin use were found to be linked to high ACE2 levels. ${ }^{24}$ Some have suggested that sustained hyperglycemia may add to the pathogenicity of SARS-CoV-2 and degree of COVID-19 illness severity by promoting the binding of the virus to ACE2 receptors in the lung. ${ }^{46}$ The viral spike protein subunit responsible for binding the receptor domain is heavily glycosylated, ${ }^{47}$ and thus may be a potential therapeutic target in COVID-19. In a non-obese diabetic mouse model, ACE2 levels in the lung are increased at baseline (thought to be due to an upregulated renin-angiotensin system), but decrease to control levels after insulin administration. ${ }^{48}$ Remarkably, elevated levels of the protease furin, that cleaves the subunits of the viral spike protein, are also associated with the development of DM, thus acting as an additional possible link between DM and COVID-19 disease severity. ${ }^{49}$

ACE2 receptors are also implicated in the physiologic derangements seen in DM, though it is not certain whether ACE2 itself directly regulates glycemic control. ${ }^{50}$ Interestingly, in the acute illness, scientists have raised the possibility that SARS-CoV-2 may cause pancreatic endocrine dysfunction by binding ACE2 receptors in the pancreas, resulting in hyperglycemia requiring insulin treatment during hospitalization. ${ }^{24}$ Thus, Cristelo et al. hypothesize that there may be a "paracrine loop" (Figure 1) that propagates increased viral binding to glycosylated ACE2 receptors in the lung by damaging pancreatic islet cells and perpetuating hyperglycemia. ${ }^{51}$ This is clinically relevant, as the damage caused to the pancreas and the resulting DM in patients with SARS has been seen to last up to 3 years. ${ }^{52}$ Similar damage has already been observed in the pancreas of patients infected with SARS-COV-2.53

It is unclear whether patients with diabetes on ACE inhibitors or angiotensin receptor blockers (ARBS) are benefited by, or are at higher risk from, these medications. It has been proposed that ACE inhibitors and ARBs increase expression of ACE2 receptors, thus possibly increasing viral entry into lung epithelium; though observational studies have not proven this. ${ }^{51,54}$ Conversely, others have hypothesized that treatment with ACE inhibitors and ARBS protects pneumocytes from lung injury, which has been demonstrated in animal models of SARS. ${ }^{55}$ Of note, the relationship between SARS-COV-2 and human ACE2 receptors is one of a number of biological mechanisms proposed for increased infectivity and worse outcomes in predisposed patients. Other proposed novel routes for SARS-CoV-2 infection include the CD147 receptor on host cells, ${ }^{56}$ sialic acids linked to host cell surface gangliosides, ${ }^{51}$ transmembrane protease serine 2, and dipeptidyl peptidase-4, the latter two being established transducers of metabolic and inflammation signaling. ${ }^{49}$ Several studies have also shown that patients with DM have a decreased forced vital capacity and forced expiratory volume in 1 second, and that this reduced pulmonary capacity may increase general susceptibility to respiratory infections like SARS-CoV-2. ${ }^{57}$

\section{Conclusion}

DM is the most prevalent non-communicable chronic disease worldwide. ${ }^{58}$ There is a large population in the USA who do not have a known DM diagnosis but have pre-diabetic or diabetic-range HbA1C noted on presentation, which can put them at risk for increased complications from COVID-19. ${ }^{4}$ DM and subsequent hyperglycemia dampen the immune response to infection, increase inflammation, and increase the risk of ICU admission in patients hospitalized with viral respiratory illness. Over one in 10 residents of the USA have been diagnosed with DM, and descriptive literature has identified even higher rates of DM in patients hospitalized with COVID-19.59,60 A number of mechanisms have been identified to explain the link between DM and morbidity from COVID-19 specifically, including a hyperinflammatory state causing coagulopathy and tissue damage, host cell surface marker regulation, decreased overall pulmonary function, and a paracrine loop model that describes viral binding in the lungs and pancreas in the setting of worsening hyperglycemia and glycosylation.

Marginalized populations and underrepresented minorities have been disproportionately affected by COVID-19 in the USA with higher rates of cases, hospital admissions, and fatalities. ${ }^{61}$ The age of onset of DM is also decreasing among minorities. ${ }^{20}$ Disparities in healthcare access cause higher rates of DM in oppressed racial groups, ${ }^{4}$ and may be contributing to the disproportionate impact of COVID-19 on African American/Black, Hispanic/Latinx, and Native American/Alaska Native patients. Advanced age populations, which have suffered higher mortality rates with COVID-19, 
also have had higher rates of DM. ${ }^{4}$ Most immediately, healthcare institutions should undertake aggressive screening for DM in all patients, and very close monitoring and control of blood glucose during hospitalization.

This outbreak should also serve as a reminder of the immense importance of targeted initiatives in public health and preventive medicine. The widespread impact of COVID-19 illustrates the importance of primary care providers, improving healthcare access, addressing social inequities, screening for comorbidities, and more intensive chronic disease management. With the significant likelihood that high acuity COVID-19 cases will continue to present in large numbers, it is also important to pursue further basic science and clinical study to better understand the mechanisms by which DM affects development and virulence of SARS-CoV-2 infection and overall outcomes. Potential future therapies may involve modulating key mediators of immunometabolism to reduce viral duration, proliferation, and severity of COVID-19 cases. $]$
1. Worldometer. Worldometer COVID-19 data. 2020. Available at: www.worldometers.info/coronavirus/ (accessed November 27, 2020)

2. Roser M, Ritchie H, Ortiz-Ospina E, Hasell J. Coronavirus pandemic (COVID-19), 2020. Available at: https://ourworldindata.org/ coronavirus (accessed October 12, 2020)

3. Horbach SPJM. Pandemic publishing: medical journals strongly speed up their publication process for COVID-19. Quant Sci Stud. 2020;1:1056-67.

4. Muniyappa R, Gubbi S. COVID-19 pandemic, coronaviruses, and diabetes mellitus. Am J Physiol Endocrinol Metab. 2020:318:736-41.

5. Capes SE, Hunt D, Malmberg K, Gerstein HC. Stress hyperglycaemia and increased risk of death after myocardial infarction in patients with and without diabetes: a systematic overview. Lancet. 2000;355:773-8.

6. Golden SH, Peart-Vigilance C, Kao WHL, Brancati FL. Perioperative glycemic control and the risk of infectious complications in a cohort of adults with diabetes. Diabetes Care. 1999:22:1408-14.

7. Furnary AP, Zerr KJ, Grunkemeier GL, Starr A. Continuous intravenous insulin infusion reduces the incidence of deep sternal wound infection in diabetic patients after cardiac surgica procedures. Ann Thorac Surg. 1999;67:352-60

8. Hansen TK, Thiel S, Wouters PJ, et al. Intensive insulin therapy exerts antiinflammatory effects in critically ill patients and counteracts the adverse effect of low mannose-binding lectin levels. J Clin Endocrinol Metab. 2003;88:1082-8.

9. Seshasai SRK, Kaptoge $\mathbf{S}$, Thompson A. The emerging risk factors collaboration. Diabetes mellitus, fasting glucose, and risk of cause-specific death. N Eng/ J Med. 2011:364:829-41.

10. Allard R, Leclerc P, Tremblay C, Tannenbaum TN. Diabetes and the severity of pandemic influenza A (H1N1) infection. Diabetes Care. 2010;33:1491-3.

11. Chan-Yeung M, XU RH. SARS: epidemiology. Respirology. 2003;8(Suppl. 1):S9-14.

12. Booth $\mathrm{CM}$, Matukas $\mathrm{LM}$, Tomlinson GA, et al. Clinical features and short-term outcomes of 144 patients with SARS in the Greater Toronto area JAMA. 2003:289:2801-9.

13. Morra ME, van Thanh L, Kamel MG, et al. Clinical outcomes of current medical approaches for Middle East respiratory syndrome: a systematic review and meta-analysis. Rev Med Virol 2018;28:e1977.

14. Wu C, Chen X, Cai Y, et al. Risk factors associated with acute respiratory distress syndrome and death in patients with coronavirus disease 2019 pneumonia in Wuhan, China. JAMA Intern Med 2020;180:934-43.

15. Yang JK, Feng Y, Yuan MY, et al. Plasma glucose levels and diabetes are independent predictors for mortality and morbidity in patients with SARS. Diabet Med. 2006;23:623-8.

16. Garbati MA, Fagbo SF, Fang VJ, et al. A comparative study of clinical presentation and risk factors for adverse outcome in patients hospitalised with acute respiratory disease due to MERS coronavirus or other causes. PLOS One. 2016;11:2-12.

17. Poissy J, Goffard A, Parmentier-Decrucq E, et al. Kinetics and pattern of viral excretion in biological specimens of two MERS-COV cases J Clin Virol 2014:61:275-8.

18. Endocrine Society. U.S. leads developed nations in diabetes prevalence, 2015. Available at: https://endocrinenews.endocrin org/U-s-leads-developed-nations-in-diabetes-prevalence/ (accessed October 12, 2020).

19. Richardson S, Hirsch JS, Narasimhan M, et al. Presenting characteristics, comorbidities, and outcomes among 5700 patients hospitalized with COVID-19 in the New York City area. IAMA. 2020;323:2052-9.

20. Boord JB, Graber AL, Christman JW, Powers AC. Practical management of diabetes in critically ill patients. Am J Respir Crit Care Med. 2001;164:1763-7.
21. Zhang JJ, Dong X, Cao YY, et al. Clinical characteristics of 140 patients infected with SARS-CoV-2 in Wuhan, China. Allergy. 2020:75:1730-41.

22. Hu Y, Sun J, Dai Z, et al. Prevalence and severity of corona virus disease 2019 (COVID-19): A systematic review and meta-analysis J Clin Virol. 2020:127:104371.

23. Huang $C$, Wang $Y, L i X$, et al. Clinical features of patient infected with 2019 novel coronavirus in Wuhan, China. Lancet. 2020;395:497-506

24. Brufsky A. Hyperglycemia, hydroxychloroquine, and the COVID-19 pandemic. J Med Virol. 2020:92:770-5.

25. Kitabchi AE, Umpierrez GE, Miles JM, Fisher JN. Hyperglycemic crises in adult patients with diabetes. Diabetes Care 2009:32:1335-43

26. Xu G, Liu B, Sun Y, et al. Prevalence of diagnosed type 1 and type diabetes among US adults in 2016 and 2017: population based study. BMJ. 2018;362:k1497.

27. Ebekozien OA, Noor N, Gallagher MP, Alonso GT. Type 1 diabetes and COVID-19: preliminary findings from a multicenter surveillance study in the U.S. Diabetes Care. 2020;43:e83-5.

28. Tatti $\mathrm{P}$, Tonolo $\mathrm{G}$, Zanfardino $\mathrm{A}$, lafusco $\mathrm{D}$. Is it fair to hope that patients with type 1 diabetes (autoimmune) may be spared by the infection of Covid-19? Med Hypotheses. 2020;142:109795.

29. Emami A, Javanmardi F, Pirbonyeh N, Akbari A. Prevalence of underlying diseases in hospitalized patients with COVID-19: a systematic review and meta-analysis. Arch Acad Emerg Med. 2020:8:e35.

30. Fadini GP, Morieri ML, Longato E, Avogaro A. Prevalence and impact of diabetes among people infected with SARS-CoV-2 J Endocrinol Invest. 2020:43:867-9.

31. Guan W, Ni Z, Hu Y, et al. Clinical characteristics of coronavirus disease 2019 in China. N Engl J Med. 2020;382:1708-20.

32. Onder G, Rezza G, Brusaferro S. Case-fatality rate and characteristics of patients dying in relation to COVID-19 in Italy. JAMA. 2020;323:1775-6.

33. Xie J, Tong Z, Guan X, Du B, Qiu H. Clinical characteristics of patients who died of coronavirus disease 2019 in China. JAMA Netw Open. 2020:3:e205619.

34. Kim M, Berger D, Matte T. Diabetes in New York City: public health burden and disparities, City of New York Department of Health and Mental Hygiene, 2007. Available at: www1.nyc.gov/assets/ doh/downloads/pdf/epi/diabetes_chart_book.pdf (accessed October 12, 2020)

35. Li B, Yang J, Zhao F, et al. Prevalence and impact of cardiovascular metabolic diseases on COVID-19 in China. Clin Res Cardiol. 2020;109:531-8.

36. Wang $\mathrm{D}, \mathrm{Hu} \mathrm{B}$, Hu C, et al. Clinical Characteristics of 138 hospitalized patients with 2019 novel coronavirus-infected pneumonia in Wuhan, China. JAMA. 2020;323:1061-9.

37. Bhatraju PK, Ghassemieh BJ, Nichols M, et al. Covid-19 in critically ill patients in the Seattle region-case series. N Engl I Med. 2020;382:2012-22.

38. CDC COVID-19 Response Team. Preliminary estimates of the prevalence of selected underlying health conditions among patients with coronavirus disease 2019 - United States, February 12-March 28, 2020. MMWR Morb Mortal Wkly Rep. 2020;69:382-6.

39. Zhou F, Yu T, Du R, et al. Clinical course and risk factors for mortality of adult inpatients with COVID-19 in Wuhan, China: a retrospective cohort study. Lancet. 2020;395:1054-62.

40. Guo W, Li M, Dong Y, et al. Diabetes is a risk factor for the progression and prognosis of COVID-19. Diabetes Metab Res Rev. 2020; 3319.

41. Kulcsar KA, Coleman CM, Beck SE, Frieman MB. Comorbid diabetes results in immune dysregulation and enhanced disease severity following MERS-COV infection. JCl Insight. 2019;4:e131774.

42. Wang S, Ma P, Zhang S, et al. Fasting blood glucose at admission is an independent predictor for 28-day mortality in patients with COVID-19 without previous diagnosis of diabetes: a multi-centre retrospective study. Diabetologia. 2020;63:2102-11.

43. Chen X, Hu W, Ling J, et al. Hypertension and diabetes delay the viral clearance in COVID-19 patients. medRxiv. 2020: doi: 10.1101/2020.03.22.20040774 [preprint].

44. Tsai S, Clemente-Casares X, Zhou AC, et al. Insulin receptor-mediated stimulation boosts $\mathrm{T}$ cell immunity during inflammation and infection. Cell Metabolism. 2018;28:922-34.e4.

45. Rao S, Lau A, So HC. Exploring diseases/traits and blood proteins causally related to expression of ACE2, the putative receptor of SARS-CoV-2: a Mendelian randomization analysis highlights tentative relevance of diabetes-related traits. Diabetes Care. 2020:43:1416-26.

46. Wrapp D, Wang N, Corbett KS, et al. Cryo-EM structure of the 2019-nCoV spike in the prefusion conformation. Science. 2020;367:1260-63

47. Roca-Ho H, Riera M, Palau V, et al. Characterization of ACE and ACE2 expression within different organs of the NOD mouse. Int J Mol SCi. 2017:18:563.

48. Fernandez C, Rysä J, Almgren P, et al. Plasma levels of the proprotein convertase furin and incidence of diabetes and mortality. J Intern Med. 2018;284:377-87.

49. Drucker DJ. Coronavirus infections and type 2 diabetes -shared pathways with therapeutic implications. Endocr Rev. 2020;41:bnaa011.

50. Yang J, Zheng Y, Gou X, et al. Prevalence of comorbidities and its effects in coronavirus disease 2019 patients: a systematic review and meta-analysis. Int I Infect Dis. 2020:94:91-5.

51. Cristelo C, Azevedo C, Marques JM, et al. SARS-CoV-2 and diabetes: new challenges for the disease. Diabetes Res Clin Pract. 2020;164:108228

52. Yang JK, Lin SS, Ji XJ, Guo LM. Binding of SARS coronavirus to its receptor damages islets and causes acute diabetes. Acta Diabetol. 2010;47:193-9.

53. Jarcho J, Ingelfinger J, Hamel MB, et al. Inhibitors of the renin-angiotensin-aldosterone system and covid-19. N Eng/ J Med. 2020;382:e102

54. Imai Y, Kuba K, Rao S, et al. Angiotensin-converting enzyme 2 protects from severe acute lung failure. Nature. 2005;436:112-6.

55. Ulrich H, Pillat MM. CD147 as a target for COVID-19 treatment: suggested effects of azithromycin and stem cell engagement. Stem Cell Rev Rep. 2020;16:434-40.

56. Fantini J, di Scala C, Chahinian H, Yahi N. Structural and molecular modelling studies reveal a new mechanism of action of chloroquine and hydroxychloroquine against SARS-CoV-2 infection. Int J Antimicrob Agents. 2020;55:105960.

57. Maddaloni E, Buzzetti R. Covid-19 and diabetes mellitus: unveiling the interaction of two pandemics. Diabetes Metab Res Rev. 2020;e33213321.

58. Selvin E, Zhu H, Brancati FL. Elevated A1C in adults without a history of diabetes in the U.S. Diabetes Care. 2009;32:828-33.

59. US Department of Heath and Human Services. Centers for Disease Control and Prevention (CDC). National diabetes statistics report 2020. Estimates of diabetes and its burden in the United States. Available at: www.cdc.gov/diabetes/pdfs/data/statistics/ national-diabetes-statistics-report.pdf (accessed November 27 2020)

60. Tenforde MW, Billig Rose E, Lindsell CJ, et al.; CDC COVID-19 Response Team. Characteristics of adult outpatients and Inpatients with COVID-19 - 11 academic medical centers, United States, March-May 2020. MMWR Morb Mortal Wkly Rep. 2020:69:841-6.

61. Kirby T. Evidence mounts on the disproportionate effect of COVID-19 on ethnic minorities. Lancet Respir Med. 2020;8:547-8. 